\title{
A STUDY OF THE SO-CALLED IMPLANTATION OF THE BACILLUS BULGARICUS *
}

\author{
ALFRED H. RAHE \\ (From the Department of Experimental Pathology, Cornell Medical College, New York City)
}

After Metchnikoff's announcement that certain lactic acid-forming bacilli found in the fermented milk Yahourth were able to check the growth of putrefactive bacteria in the intestines, in all the articles published in attempted confirmation of his claims, the methods used to identify the bacillus are inadequately described. This work was undertaken with the purpose of determining, in the light of a better understanding of the cultural peculiarities of the bacillus bulgaricus, whether or not a true implantation took place.

In the human intestine there occur normally bacilli that culturally and morphologically resemble this bacillus very closely. The group of organisms, which includes the bacillus bulgaricus, is distinguished from other groups of bacteria by the ability of its members to grow in media containing considerable amounts of acid; because of this characteristic, these bacilli are called aciduric or acid-tolerant organisms. In a previous paper ${ }^{1}$ the writer was able to show that, contrary to the usual statement, these bacteria grow luxuriantly in an ordinary laboratory medium, viz., unneutralized meat-peptone broth containing glucose or other suitable carbohydrate, and that agar prepared from this broth, with or without the addition of 0.2 percent of sodium oleate, is an excellent solid medium. It was also shown that while the bacillus bulgaricus, from its cultural and perhaps biochemical properties, also belongs to this group of sugar fermenting, acid-tolerating organisms, it differs from the bacteria of the type of the bacillus acidophilus in failing to ferment maltose. Some strains do not attack saccharose.

Rrview OF THE Literature

Bertrand and Duchacek ${ }^{2}$ mention the failure of this organism to ferment maltose, but do not give the nature of the medium used. On the other hand

* Received for publication December 3, 1914:

1. Jour. Infect. Dis., 1914,15 , p. 141.

2. Ann. de 1'Inst. Pasteur, 1909, 23, p. 402.

3. Rev. méd. de la Suisse romande, 1905,25 , p. 714.

4. Compt. rend Soc. de Biol., 1906, 60, p. 558. 
Grigoroff and Cohendy; equally indefinite as to medium, claim an opposite result. This contradiction in evidence leads to the suspicion that faulty identification nay have led, in at least some instances, to erroneous conclusions as to the implantation and survival of this bacillus in the intestine. Cohendy claims to have demonstrated that the organism becomes established in the intestine in eight days and survives for twelve days or less after feeding has been stopped. According to Belanowsky ${ }^{5}$ the bacillus becomes adapted to the human

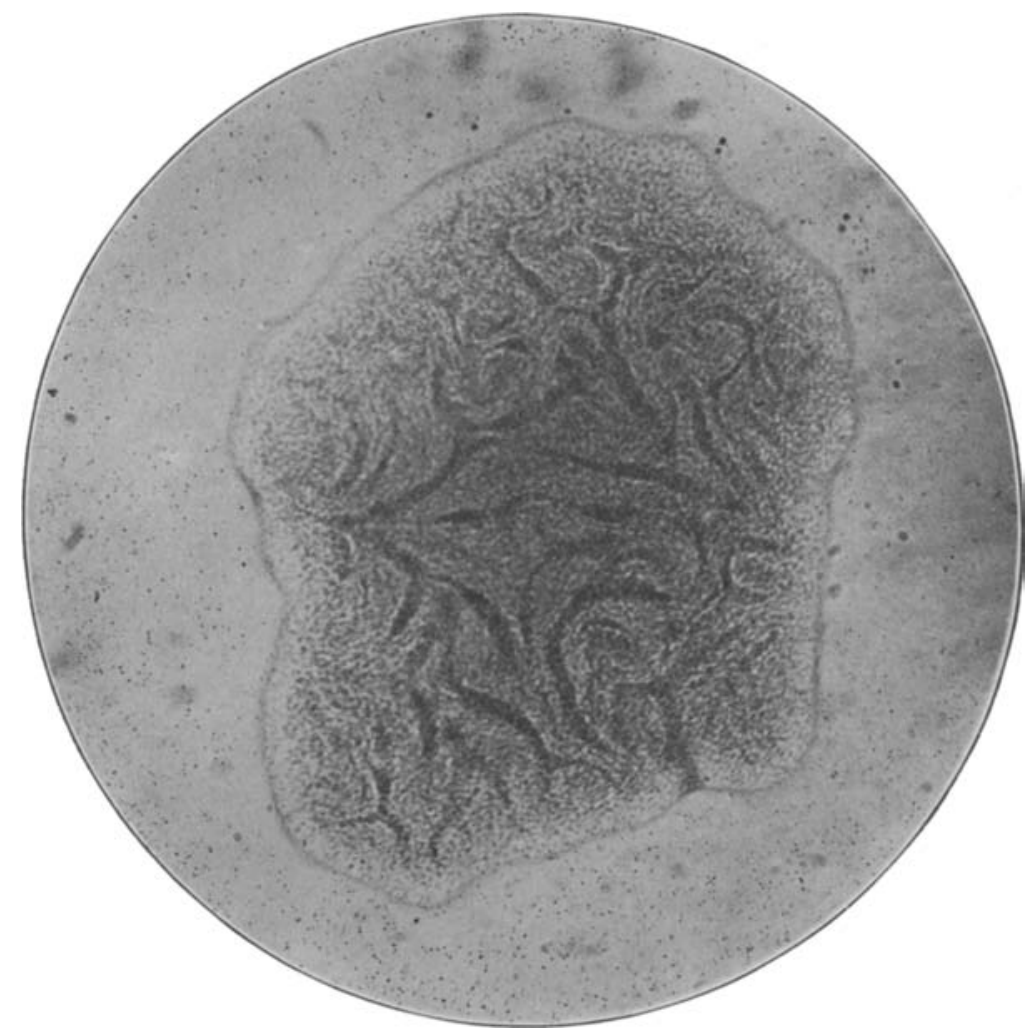

Fig. 1.-An atypical colony of the bacillus bulgaricus. No projections. $\times 60$.

intestine and survives for varying periods. Distaso and Schiller" fed the organism to rats and concluded that it is impossible to force the growth of a foreign organism in the intestine. Herter and Kendall fed to monkeys Bacillac, a commercial fermented milk in which the bacillus did not occur in pure culture. The organism was recovered from the stools on the fifth day after feeding commenced. After fourteen days of exclusive feeding with this milk the organism was found in almost pure culture in the duodenum and jejunum, in

5. Ann. de l'Inst. Pasteur, 1907, 21, p. 991.

6. Compt. rend. Soc. de Biol., 1914, 66, p. 243.

7. Jour. Biol. Chem., 1908, 5, p. 293. 
less numbers in the cecum, and hardly at all in the colon and rectum. In a personal communication to the writer, Dr. Kendall has described the method of isolation used in his investigation. A small amount of feces was seeded into milk and pure cultures obtained by repeated transfer in this medium. The organism was recognized by its complete parasitization to milk.

It seems certain that Herter and Kendall succeeded in isolating the organism ingested, but in the case of other workers the lack of

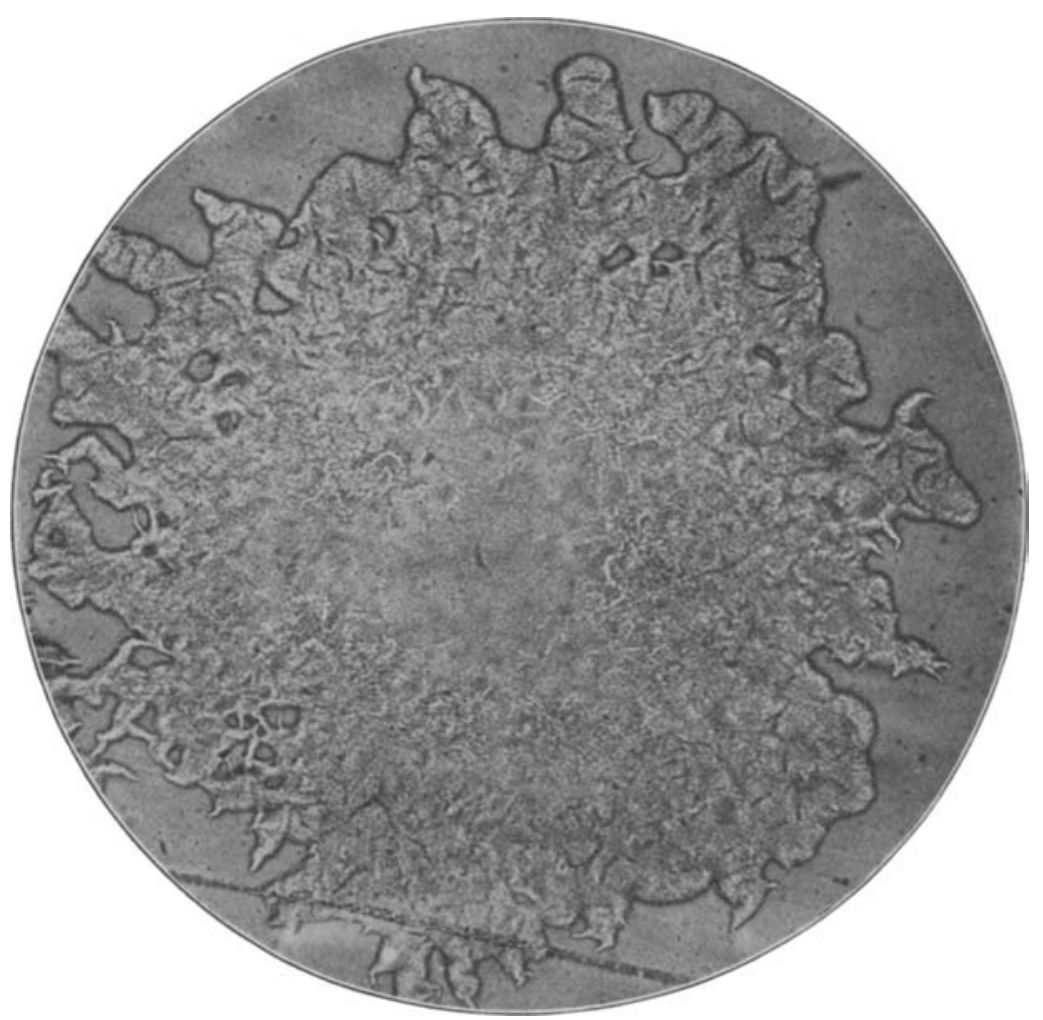

Fig. 2.-Colony of intestinal aciduric bacteria simulating that of the bacillus bulgaricus. $\times 90$.

cultural detail leaves one in doubt as to the correctness of their findings.

For the identification of the bacillus bulgaricus colony formation, morphology and quantitative estimation of acid production are insufficient. The colonies are usually described as resembling those of the bacillus anthracis in their loose texture, irregular outline, and forma- 
tion of projections. While this so-called characteristic form is frequent, a more regular form, one that resembles the typical form only in its fissured surface, is also common (Fig. 1). During the course of this work there often occurred on the plates, made from the entichment tubes which had been planted with feces, colonies of aciduric bacteria normal to the intestine that very closely resembled those of the bacillus bulgaricus (Figs. 2 and 3). During the period of ingestion

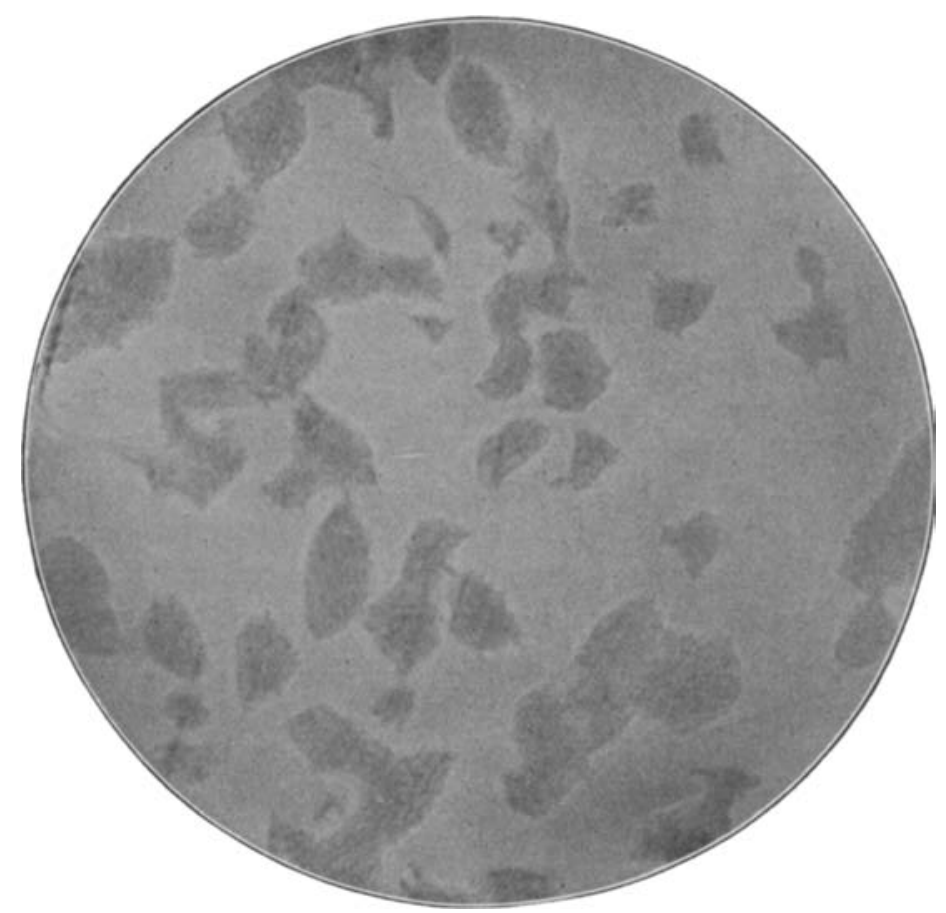

Fig. 3.-Colonies of the bacillus acidophilus with projections. About $\times 6$.

mixed colonies, composed of the bacillus bulgaricus and intestinal acid-tolerant bacilli, were common. In staining and shape the bacillus bulgaricus is identical with the bacillus acidophilus.

While some strains of the Bulgarian bacillus are capable of a high acid production, others do not produce so great an acidity as that formed by some strains of the bacillus acidophilus. The inability of the bacillus bulgaricus to utilize maltose constitutes its essential difference from the intestinal aciduric bacteria that coagulate milk. 
In this investigation pure forty-eight-hour cultures of the bacillus bulgaricus were ingested in the quantities indicated on the charts. Stools were collected at regular daily intervals and treated as follows: Within a few minutes after the passage of the movement a representative sample of $0.5 \mathrm{gm}$. was accurately weighed and emulsified in 50 c.c. of normal salt solution. This suspension was shaken for three minutes and the grosser particles allowed to settle. A series of dilutions was prepared and 0.5 c.c. of each dilution was seeded into tubes containing 10 c.c. of milk having an acidity of plus 2.5 percent normal lactic acid. Milk, acidified or not, has been used as an enrichment medium by practically all of the workers in this field. After six days' incubation at $37 \mathrm{C}$. a loopful from each milk tube was streaked on the surface of hardened and dried meat-peptone-oleate-glucose agar. After forty-eight hours' incubation likely colonies were fished and maintained in the unneutralized glucose broth.

On the plates from the enrichment tubes there were, as might be expected, mixed colonies composed of intestinal aciduric organisms and the bacillus bulgaricus, but the latter could not be distinguished as such under the microscope. In most cases cultures from such mixed colonies gave an acidity in milk nearly as great as that of the pure cultures of the organism ingested. Growth in maltose broth showed the presence of intestinal organisms and plates made from the primary cultures in glucose broth invariably showed colonies of both organisms, while plates from the maltose broth showed colonies of bacilli only of the acidophilic type. Occasionally in the mixed cultures in milk the intestinal organism predominated and the acidity did not rise above plus 17.0 percent normal in six days.

An attempt was made not only to determine whether the organism ingested could survive in the intestine but also to determine to what extent it was present. To that end, in addition to the monkeys, human subjects presenting varying intestinal conditions were chosen. In all of the experiments that follow the culture was taken just before meals.

Subject A, female, 32 years old, in the third or fourth month of pregnancy. The stools were typically putrefactive. There was only a slight constipation. The average daily diet of Subjects $A$ and $C$ was as follows : fruit $407 \mathrm{gm}$., bread $147 \mathrm{gm}$., cereal $158 \mathrm{gm}$., meat $230 \mathrm{gm}$., vegetable $280 \mathrm{gm}$., cane sugar 27 gm., milk 100 c.c., dessert (bread pudding, tapioca, blanc-mange) $142 \mathrm{gm}$.

Chart 1 shows that the organism appeared in the stools of Subject A for the first time on the eighth day. For the period during which broth culture was fed the curve reaches its greatest height on the tenth day and this point is not passed during the increased ingestion in the incompletely recorded period of broth culture feeding that follows. With the milk culture the excretion reaches its greatest height, but 

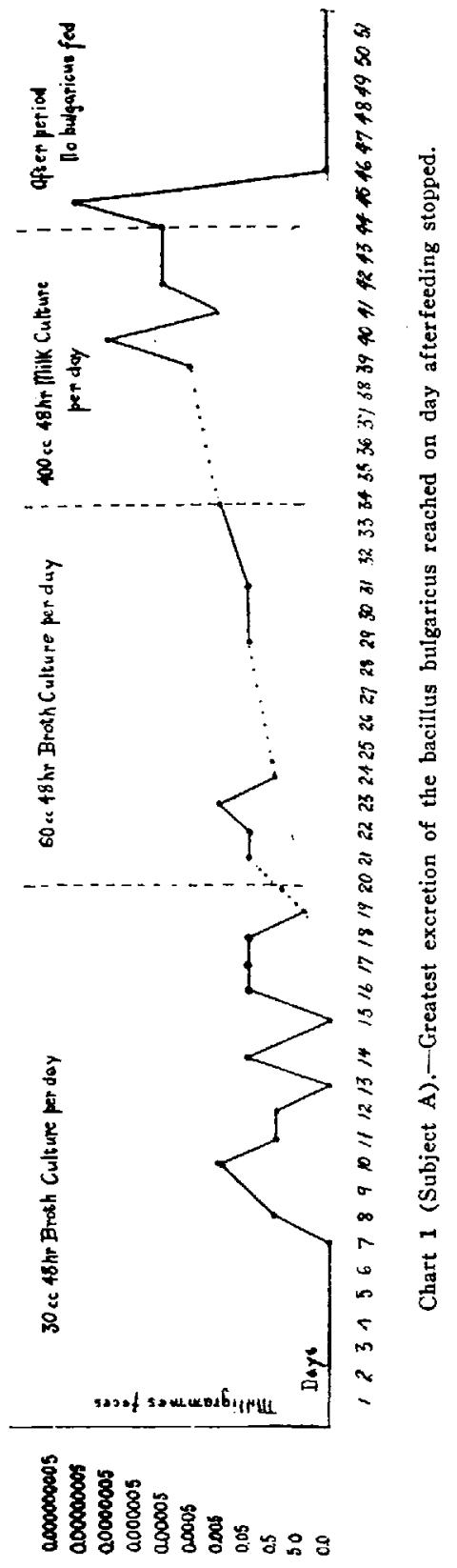
the greatest elimination occurs on the day after feeding was stopped. The bacillus disappeared from the stools on the following day.

Stbject B, male, aged 53. He subsisted both before and during the experiment entirely upon vegetable food. His stools were dry, almost odorless, and characterized by their high content of aciduric bacteria. One liter of a fortyeight-hour milk culture was taken per day. The bacillus bulgaricus appeared in the feces for the first time on the fifth day and the greatest excretion was reached on the sixth day. After its first appearance the organism was absent from two consecutive stools on two occasions. It maintained its level for one day after the feeding stopped and disappeared on the third day after the last ingestion.

The organism was fed to Subject B in enormous numbers. Altho diet and intestinal conditions were such as would be expected to favor its development, and the bacillus appeared in the stools three days

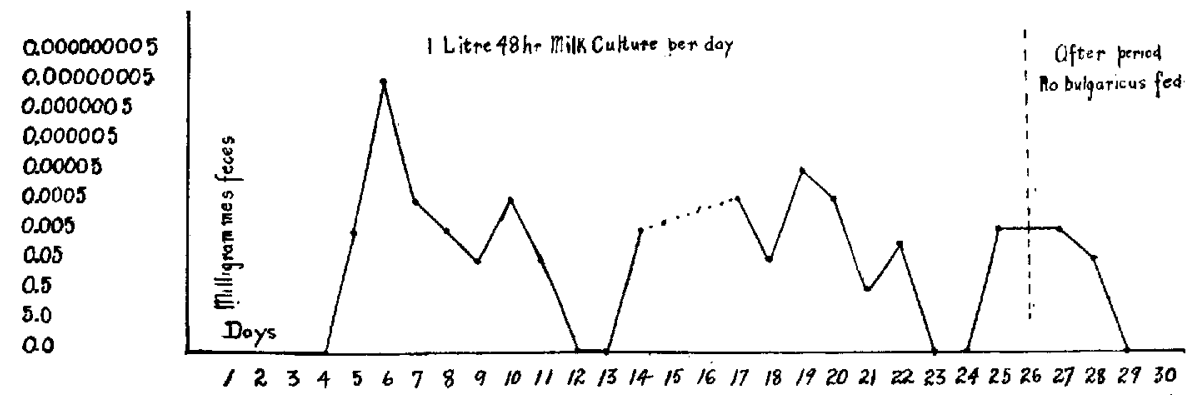

Chart 2 (Subject B).-Ingested organism absent on third day after feeding stopped.

earlier than in the preceding experiment, it survived but one day longer.

Subject C, male, 32 years old. The diet was of the same quantity and composition as that of Subject $A$. The feces were moderately putrefactive. The organism appeared on the second day following the first ingestion and survived for the same length of time as in the case of Subject $B$. As in the other two subjects, this bacillus disappeared completely from the stools within a few days after the ingestion of the culture was stopped and was not recovered again, altho the examinations were continued daily for a week. In one instance during the feeding of the culture the bacillus was absent from three consecutive stools.

Neither in this nor in the preceding experiments did the disappearance of the organism occur at times when several stools were passed during two days. Their absence must have been due to some other cause than a sudden elimination. Either the bacillus died off rapidly 

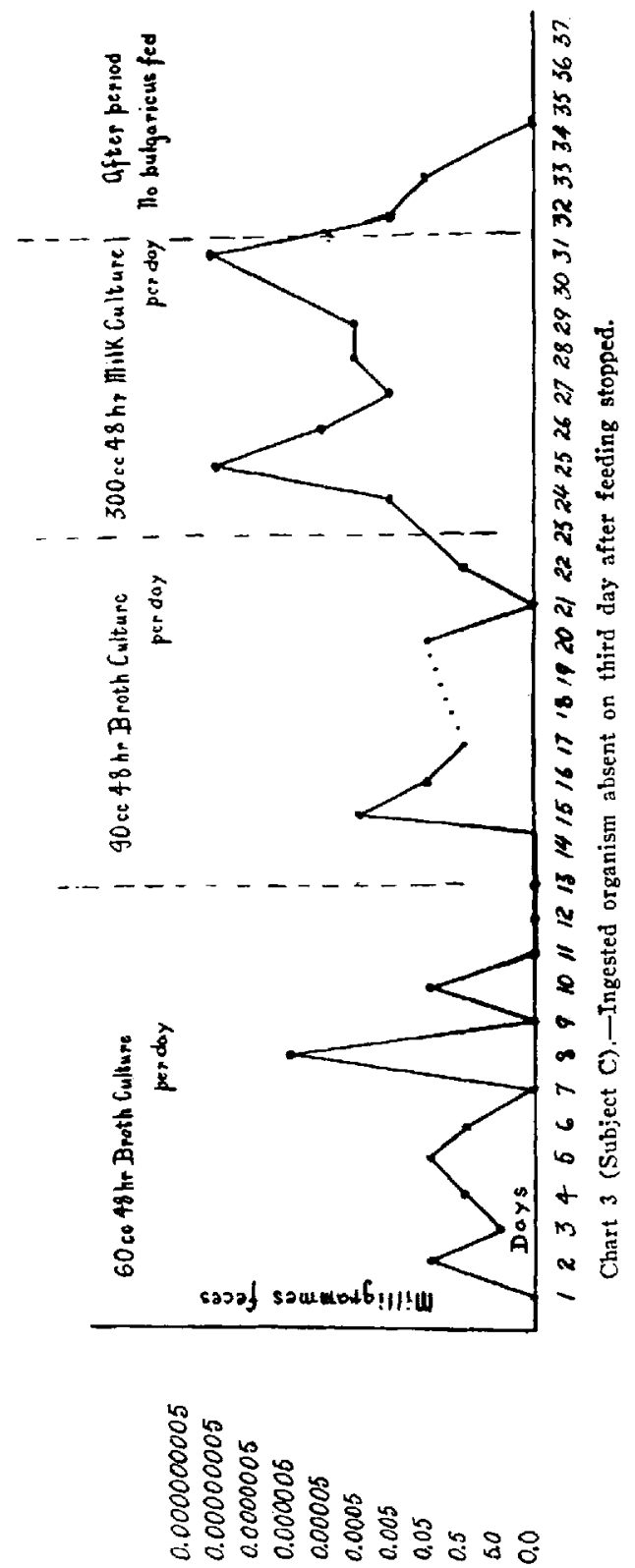
or it was retained in the upper levels of the digestive tube and passed into the feces in numbers too small to be detected.

Subject W., female, 25 years old, had been taking the bacillus bulgaricus in tablets for several months. For eight weeks during which this subject received 60 c.c. of broth culture per day the stools were examined at irregular intervals. The organism was found present in nearly all of the stools tested and survived eight days after feeding stopped.

Altho in the above experiments the bacillus bulgaricus disappeared from the feces soon after ingestion stopped, it has not been proved that the organism does not survive for a longer period in the small intestine. It is possible that an organism might be able to adapt itself to the conditions prevailing in the duodenum and jejunum, but be unable to survive in the lower intestine, and so be absent from the stools.

In order to determine if such survival occurred, small Rhesus monkeys that had been fed the bacillus bulgaricus were killed at different intervals after the feeding was discontinued. Five-tenths of a gram of the contents of the different regions of the intestine were treated in the same way as the feces of the human subjects. The monkeys were fed twice a day and received the following average diet: one banana, $99 \mathrm{gm}$. boiled rice, one apple, $58 \mathrm{gm}$. bread, $62 \mathrm{gm}$. cabbage.

Monkey 1 was given fifty lactose tablets per day, each of which contained approximately 2,000 bacilli. The tablets were prepared as follows: after a heavy broth culture was centrifugalized, the deposited organisms were suspended in a very small amount of salt solution, and with lactose were formed into tablets. Fresh forty-eight-hour cultures were used and the tablets fed just before meals. After the thirteenth day the feeding was stopped and the animal killed on the day following. The bacillus bulgaricus was recovered in fairly large numbers from the jejunum, but not from the other parts of the digestive tube and feces.

Monkey 2 was fed 300 c.c. of forty-eight-hour milk culture per day for six days and cultured seven hours after the last feeding. The organism was found in large numbers in the duodenum, jejunum, ileum, and feces, and very scantily in the colon.

Monkey 3 received the same amount of culture for the same length of time as Monkey 2, but the feeding was stopped seven days before death. The bacillus was recovered in small numbers and only from the duodenum.

This series of experiments, while not complete, serves to show that the organism may survive in the small intestine after it has disappeared from the lower digestive tract and feces, tho it is probable that this survival is not permanent. Table 1 gives the results of these experiments. 
Altho it is probable that the bacillus bulgaricus once flourished in the intestine of a warm blooded animal, as its preference for body temperature shows, the facts brought out in this investigation indicate that it is no longer capable of developing in the lower part of the digestive tube. Its absence from the feces, unless previously ingested in great numbers, is especially interesting in view of the fact that Hastings and Hammer ${ }^{8}$ have discovered an organism resembling the Bulgarian bacillus in milk, butter, and cheese. It is probable that their bacillus was an intestinal organism, but, since these authors do not state whether it attacks maltose, it is probable that they were dealing with an exceptionally active strain of the bacillus acidophilus.

TABLE 1

The Survival cf the Bacillus Bulgaricus in the Intestines of Monkeys

\begin{tabular}{|c|c|c|c|c|c|c|c|c|}
\hline Monkey & Fed & $\begin{array}{l}\text { Perlod of } \\
\text { Ingestion }\end{array}$ & $\begin{array}{c}\text { Time } \\
\text { After } \\
\text { Last } \\
\text { Ingestion }\end{array}$ & $\underset{\text { denum }}{\text { Duo- }}$ & $\begin{array}{l}\text { Jeju- } \\
\text { num }\end{array}$ & Ileum & Oolon & F'eces \\
\hline $1 \ldots \ldots$ & Fifty tablets per day....... & 13 days & $24 \mathrm{hrs.}$ & - & + & - & 一 & 一 \\
\hline $2 \ldots \ldots$ & 300 c.c. 48 -hr. milk culture.. & 6 days & $7 \cdot \mathrm{hrs}$. & + & + & + & + & + \\
\hline $9 \ldots \ldots$ & 800 c.c. 48 -hr. milk culture. & 6 days & 7 days & + & 一 & - & 一 & - \\
\hline Control & Not fed................. & $\ldots \ldots \ldots$ & $\ldots \ldots \ldots$ & 一 & - & - & - & 一 \\
\hline
\end{tabular}

The sign + denotes the presence of the bacillus bulgaricus; the sign $\cdots$ denotes absence.

The mere recovery of an organism from the feces several days after the feeding has stopped does not prove that it has become adapted to the intestine, and, while the evidence developed in this investigation does not exclude the possibility of a slight multiplication of the bacillus bulgaricus in the lower part of the human digestive tract, it is obvious that nothing resembling a true implantation took place.

The charts show that the point of greatest excretion of the ingested organism is the same for all three subjects and represents an elimination of at least twenty million organisms per milligram feces. The average daily excretion for Subjects $A, B$, and $C$ was about one million organisms per milligram.

Torrey, ${ }^{9}$ in an investigation of the fecal flora of typhoid patients on a high calory diet, found that the excretion of bacteria of the type of the bacillus acidophilus frequently reached one million organisms per milligram feces and in one instance was six times that amount.

8. Univ. of Wis. Agric. Exper. Sta. Research Bull. No. 6, 1909, p. 195.

9. Jour. Infect. Dis., 1915, 16, p. 72 . 
In the present investigation intestinal aciduric organisms were frequently encountered at very high dilutions of the feces and it is probable that their excretion paralleled if it did not exceed that of the bacillus bulgaricus, notwithstanding the enormous ingestion of the latter.

$$
\text { CONCLUSIONS }
$$

The bacillus bulgaricus is an organism readily distinguished from the intestinal aciduric bacteria.

The evidence indicates that this bacillus cannot become adapted to the human lower intestine.

The experiments with monkeys show that the bacillus bulgaricus is capable of an apparently limited survival in the upper intestine of these animals. 